\title{
Studying Shocked Material Dynamics with Ultrafast X-rays
}

R.L. Sandberg, ${ }^{1}$ C. Bolme, ${ }^{1}$ K. Ramos, ${ }^{1}$ Q.McCulloch, ${ }^{1}$ R. Martinez, ${ }^{1}$ V. Hamilton, ${ }^{1}$ T. Pierce, ${ }^{1}$ M. Greenfield, ${ }^{1}$ S.McGrane, ${ }^{1}$ J.L. Barber, ${ }^{1}$ B. Abbey, ${ }^{2}$ A. Schropp, ${ }^{3}$ F. Seiboth, ${ }^{3}$ P. Heiman, ${ }^{4}$ B. Nagler, ${ }^{4}$ E. Galtier, ${ }^{4}$ E. Granados ${ }^{4}$

1. Los Alamos National Laboratory, PO Box 1663, Los Alamos, NM 87545

2. Department of Physics, University of La Trobe, Rm 419, PS1, Melbourne, Victoria, Australia

3. Institute of Structural Physics, Technische Universitat Dresden, D-01062 Dresden, Germany

4. SLAC National Accelerator Laboratory, 2575 Sand Hill Road, Menlo Park, CA 94025, USA Author e-mail address: sandberg@lanl.gov

The response of micron-scale inhomogeneities dictates the overall dynamic, structural and chemical response of many materials. Of particular interest is the response of micron scale voids. It is believed that such micron scale voids are responsible for the nucleation of damage leading to structural failure in metals and to initiation of detonation in explosive material under high strain-rates. A critical step towards developing safer, stronger, and longer lasting materials in a range of applications from energy to defense requires understanding the dynamic response of these inhomogeneties on the micron-scale.

X-rays are particularly well suited for micron-scale materials studies due to their short wavelength and ability to penetrate bulk materials. Combining this ability with the intense ultrafast pulses from X-ray free electron lasers such as SLAC’s Linac Coherent Light Source (LCLS) provides an ideal system for studying material response under extreme conditions such as impact, high load, or other non-equilibrium processes. Here we demonstrate this groundbreaking ability by showing sub-micron single-shot X-ray imaging of laser-shocked materials performed at the Materials in Extreme Conditions (MEC) hutch at the LCLS in January 2014. We imaged shock wave interactions with 10-micron voids in two single crystal materials: lithium floride (LiF) and the explosive pentaerythritol tetranitrate (PETN) with near $100 \mathrm{~nm}$ resolution. Shock wave interactions with voids in explosive are of particular interest due to the prevailing theory that detonation initiation is caused by void collapse.

Fig. 1a shows the experimental setup at the MEC hutch for these studies. The LCLS beam was tuned to $6 \mathrm{keV}$ and focused through a set of beryllium compound refractive lenses (CRLs)[1] to focus the beam down to near $100 \mathrm{~nm}$ as verified through ptychographic imaging of the focused beam as demonstrated previously [2,3]. The $\sim 150$ micron thick single crystal samples (either LiF or PETN) were placed at a variable distance (about $5 \mathrm{~cm}$ ) behind the focus which allowed for an adjustable spot size on the sample (2-100 microns) depending on the focus to sample distance. Several detectors were used in these experiments to capture either the low angle coherent scattering (coherent diffractive imaging or Gabor holography) or the higher angle Bragg X-ray diffraction (XRD). For the low angle scatter, several detectors were available in order to optimize dynamic range, sensitivity, and pixel size including an Andor direct detection CCD, an FLI phosphor coupled CCD, and a 1x2 CS-PAD. These detectors were placed $4.1 \mathrm{~m}$ behind the samples. For the higher angle XRD a $4 \times 4$ array of CS-PAD detectors were used. Due to the brilliant ( $10^{12}$ photons) coherent pulses with 50 fs pulse duration, the laser shock dynamics were captured with no blur in a single pulse. The shock was driven perpendicular to the XFEL beam by focusing down the two arms of the $10 \mathrm{~Hz}$ glass drive laser (up to $35 \mathrm{~J}$ per pulse at 532 $\mathrm{nm}$ ) into a thin ablation layer consisting of a polyimide (25 microns) covered with $\sim 100 \mathrm{~nm}$ of aluminum. Shock pressures ranged from a few GPA up to 10 GPA as measured with line resolved laser 
velocity interferometer system for any reflector (VISAR). A silicon channel cut monochromator was also used on some shots to narrow the XFEL bandwidth. Fig1 b and c show respectively a Gabor hologram of a collapsing void in PETN and the simultaneous dynamic XRD. These images show the two-wave structure of the shock (plastic and elastic wave) and Bragg shifting and broadening due to lattice distortion. Fig. 2 shows a series of four voids before and during the shock loading with increasing time delay between the drive laser and XFEL image.

\section{References:}

[1] F. Seiboth, et al., J. Phys. Conf. Ser., vol. 499, no. 1, p. 012004, Apr. 2014.

[2] A. Schropp, et al., Sci. Rep., vol. 3, Apr. 2013.

[3] A. Schropp, et al., SPIE Optical Engineering + Applications, 2013, p. 88490R.

Figure 1. a) Schematic of the experimental setup showing the focused 6keV LCLS beam illuminating the laser-machined voids in the single crystal sample with the $20 \mathrm{~ns}$ glass laser (532 $\mathrm{nm}$ ) beam driving a shock wave perpendicular to the XFEL beam; b) single-shot X-ray image of a the two-wave shock structure interacting with the 10 micron void in a PETN single crystal; c) Dynamic X-ray diffraction pattern taken simultaneously with the image showing Bragg peak shifting and broadening due to lattice compression.

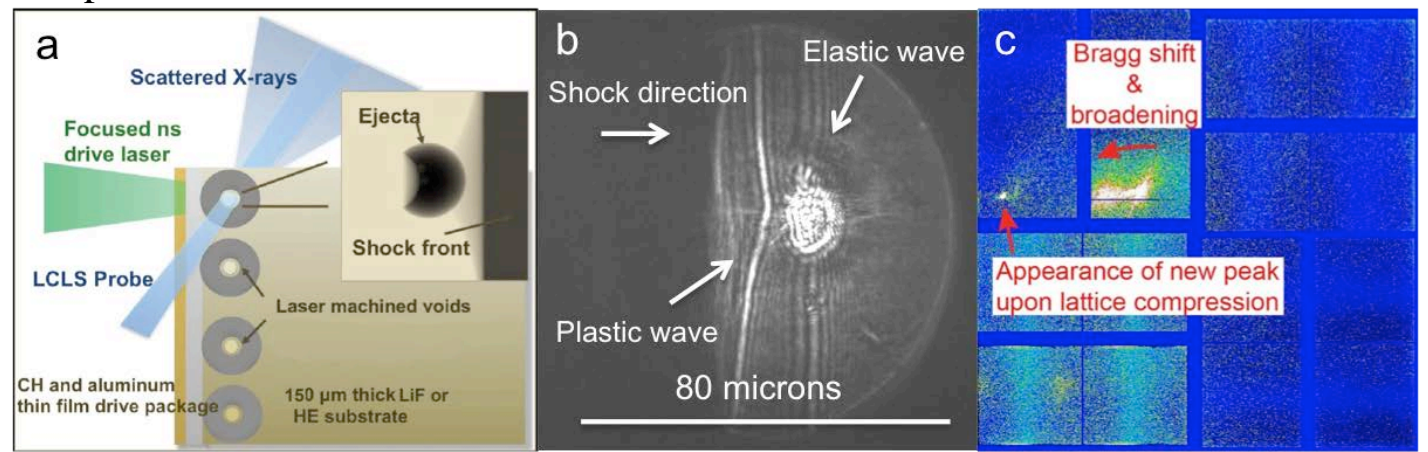

Figure 2. A montage of preliminary images comparing the static (top) and dynamic (bottom) single pulse images of four 10 micron diameter voids in $150 \mathrm{~nm}$ thick PETN at different delays with respect to the laser drive. The shock drive surface is at the top with the shock wave propagating towards the bottom. These images were taken on the FLI detector with the detector placed $4.1 \mathrm{~m}$ behind the samples.

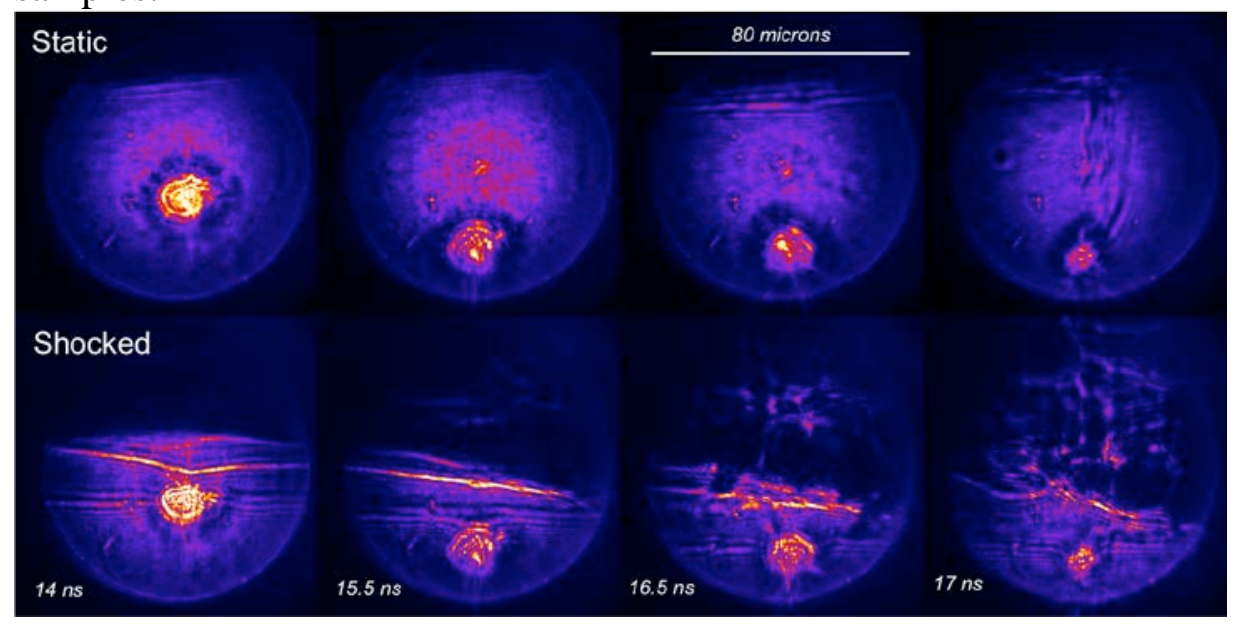

